Meta

Journal des traducteurs

Translators' Journal

\title{
Terminologie et communication
}

\section{Claire Chénard-Nantel et Noëlle Guilloton}

Volume 29, numéro 2, juin 1984

URI : https://id.erudit.org/iderudit/002226ar

DOI : https://doi.org/10.7202/002226ar

Aller au sommaire du numéro

Éditeur(s)

Les Presses de l'Université de Montréal

ISSN

0026-0452 (imprimé)

1492-1421 (numérique)

Découvrir la revue

Citer cette note

Chénard-Nantel, C. \& Guilloton, N. (1984). Terminologie et communication.

Meta, 29(2), 215-217. https://doi.org/10.7202/002226ar d'utilisation que vous pouvez consulter en ligne.

https://apropos.erudit.org/fr/usagers/politique-dutilisation/ 


\section{TERMINOLOGIE ET COMMUNICATION}

Dans la conférence inaugurale,* Pierre Bourgault (Université du Québec à Montréal) s'est attaqué directement aux problèmes actuels de communication. Nous sommes possédés d'une rage de communiquer; nous communiquons souvent l'accessoire plutôt que l'essentiel; nous sommes surchargés d'information. En résumé, maîtriser la langue, c'est obtenir pouvoir et liberté. Qui plus est, selon $M$. Bourgault, la communication est une question de vie ou de mort.

Le lendemain, nous sommes entrés dans le vif du sujet, en traitant de la terminologie et de son efficacité dans la communication. Selon Rotislav Kocourek (Université Dalhousie), il existe plusieurs critères qui peuvent déterminer l'efficacité d'un terme : 1) définition spécialisée, systémique et adéquate (rapport entre sens et référent), 2) définition fixée par l'usage, 3) emploi du terme d'une manière non ambiguë ou indication des cas de polysémie et réduction de la synonymie, 4) motivation acceptable sans interférences émotives, 5) acceptabilité langagière, entre autres la conformité graphique, 6) correspondance au type de formation lexicale acceptée (dérivation, racine), 7) facilité phonique et de mémorisation, 8) internationalité, 9) concision.

A suivi un exposé de Boyd Davis (University of North Carolina) au sujet du foisonnement terminologique aux Êtats-Unis. Exemples à l'appui, Mme Davis a démontré la prolifération de termes qu'on peut retrouver dans la langue écrite en s'inspirant, entre autres, des pages jaunes des annuaires de six régions des États-Unis. La notion de "Laundromat", par exemple, se trouve exprimée de maintes façons ("Launderette" "Coin-operated Laundries" "Launder Center", etc.), selon la vocation de l'établissement ("Laundry \& Dry Cleaning Center", "Clothing Care Center") et d'après une approche marketing ("Clean and Fresh ", "Kwik-Wash", etc.) Elle a aussi souligné la profusion de syntagmes nominaux qui provoque souvent de la confusion. Aux États-Unis, on encourage la création de termes concurrents tout en dénonçant malgré tout les formes fautives.

Nous avons ensuite pu profiter de l'expérience de Suzanne Bougie-Lauzon, responsable de la francisation de Transport Brazeau et coordonnatrice du Comité interentreprises de terminologie de transport routier. Tout a été mis en oeuvre pour franciser cette entreprise pourtant déjà francophone : affichage, distribution d'ouvrages de référence, formation de réceptionnistes, slogans, concours linguistiques, nomination d'agents de changements, bref du français omniprésent, du travail sur l'inconscient collectif.

Puis il a été question de la diffusion de la terminologie par les médias. Diane Dontigny (revue

* Colloque organisé par la Société des traducteurs du Québec et par l'Office de la langue française, 13-15 février 1984, Montréal. 
Québec Science) a fait état de l'envahissement du monde scientifique par les publications américaines et de la difficulté que l'on éprouve à se procurer la terminologie française dans les domaines de pointe, les lancements de la NASA par exemple. Les scientifiques sont de plus en plus conscients des problèmes terminologiques et font un effort pour utiliser des termes justes. Québec-Science essaie en outre de doser la quantité de nouveaux termes et de les définir au fur et à mesure dans les articles afin de suivre la politique de vulgarisation de la maison.

Dans le secteur de la publicité, Gilles Tremblay (Langagerie inc.) a exposé les modes de formation des néologismes et a montré comment les termes publicitaires ou créés par la publicité influent sur la langue courante.

En ce qui concerne la diffusion de la terminologie par les documents de l'entreprise, Pierre Gagnon (Westinghouse) a parlé de la fierté que ressentaient les employés à travailler avec des outils en français ainsi que de la difficulté à travailler avec des documents normatifs plus ou moins bien traduits.

La francisation de l'informatique pose certains problèmes qui ne sont pas insolubles cependant, comme l'a expliqué Patricia Labrie (planification, section française, IBM). Il peut être assez aisé de traduire des manuels d'utilisation d'appareils, mais lorsqu'il faut traduire des progiciels ou des informations écran, la francisation prend une tout autre dimension. En effet, avant de concevoir un produit, les ingénieurs doivent vérifier si celui-ci est appelé à être traduit et le structurer de façon qu'il soit exploitable en français. Ensuite, la traduction proprement dite est effectuée, suivie de la validation du produit francais en laboratoire. Les services de traduction d'IBM France et d'IBM Canada se partagent les traductions, uniformisent la terminologie selon le pays qui est le plus avancé dans le domaine, et adaptent selon la réglementation en vigueur. IBM pense d'ailleurs commercialiser un outil de traduction pour l'information écran.

La deuxième journée a été consacrée à l'examen des moyens et des solutions, puis à la prospective.

Les textes normatifs de nature juridique ne sont-ils pas des moyens de diffusion de la terminologie ? Antoni Dandonneau (Commission des valeurs mobilières du Québec) a montré que la loi et le règlement contribuent à accréditer la légitimité des termes qu'ils emploient, même si ces termes, dont le monde du droit - et parfois le public - est " imprégné ", sont souvent fautifs. Pour déloger ces termes, il faut changer la loi. La nouvelle Loi sur les valeurs mobilières en est un excellent exemple, et la terminologie correcte qu'elle contient se répand de plus en plus, tandis que l'ancien vocabulaire tombe dans l'oubli. Cependant, l'État devrait manifester plus vigoureusement sa volonté de francisation, en imposant dans tous les textes de loi une terminologie de qualité.

Pour améliorer la qualité du français parlé et écrit de ses professeurs et de ses élèves, l'École québécoise du meuble et du bois ouvré (Cégep de Victoriaville) a réalisé " l'intégration des langues de spécialité dans la formation professionnelle " qu'elle donne en production de meubles, rembourrage, fabrication de gabarits et de prototypes, dessin technique et conception de mobilier. Richard Côté, professeur, et Reynald Binette, terminologue, ont expliqué que, dans ce contexte, le défi à relever consistait à fournir aux enseignants et aux élèves une contrepartie française aux manuels de formation anglo-américains qui soit fidèle aux normes et à la technologie nord-américaine. Un tel projet posait deux grands problèmes : 1'uniformisation de la terminologie et la technique de rédaction. Avec l'appui de l'OLF, M. Binette a rédigé un "Vocabulaire du rembourrage", qui a servi à $\mathbf{M}$. Côté pour la rédaction de son manuel. Quatre autres vocabulaires ont suivi, et un sixième est en cours, ainsi qu'un autre manuel. L'École du meuble est maintenant le foyer d'une grande activité terminologique qui rayonne dans toute l'industrie et dont le succès à long terme dépend de l'engagement des professeurs et des élèves, futurs membres des divers corps professionnels.

Madame Hélène Cajolet-Laganière a ensuite traité du "rôle des producteurs de documentation technique dans la diffusion de la terminologie ". La documentation technique et scientifique peut être produite par l'entreprise, par ses clients et fournisseurs, ou il peut s'agir de documentation de référence (normes, ouvrages techniques, etc.). Les rédacteurs techniques, qui occupent généralement le rôle de cadres intermédiaires dans l'entreprise, doivent trouver la terminologie existante, l'adapter, la compiler, la diffuser et l'implanter. Ils rencontrent cependant plusieurs obstacles : méconnaissance de la terminologie, contraintes de temps et d'argent, et surtout manque d'intérêt. La "volonté politique "et la "fierté du travail bien fait "sont en effet indispensables à l'utilisation par les producteurs de documents techniques d'une terminologie française uniformisée, tout spécialement dans les secteurs-clés de l'économie de demain.

Quel est donc le rôle des publications terminologiques (lexiques, vocabulaires, fiches, etc.)? Charles Dupont (Air Canada) a tout d'abord traité de leurs rôles sociaux généraux : prouver l'existence de la terminologie dans les domaines techniques et scientifiques, affirmer l'identité culturelle et linguistique d'un groupe, appuyer les interventions de l'État en matière de langue. Leurs rôles sociaux spécifiques consistent à : permettre la communication entre grands usagers des langues de spécialité, faciliter la vulgarisation de la terminologie, être un instrument pédagogique, être un moyen de pression, et appuyer l'édition. Les rôles linguistiques généraux de ces publications sont les suivants : répertorier des terminologies propres à des domaines techniques ct scientifiques; préserver le caractère idiomatique des langues de spécialité ; participer au développement de la langue des sciences et des techniques au même rythme que leur évolution. Quant à leurs rôles linguistiques spécifiques, ils sont, toujours d'après $M$. Dupont, d'analyser la terminologie propre à un groupe organisationnel, de modifier des habitudes langagières, de contribuer à l'uniformisation de la terminologie et d'orienter l'usage. 
Après avoir rappelé, de son double point de vue d'informaticien et d'utilisateur, ce qu'est une banque de terminologie, Benoît Thouin (Université d'Ottawa) a exposé les conditions d'efficacité des banques de terminologie : conditions générales, conditions quant à leur contenu, conditions portant sur le matériel (ordinateur central et réseau de communications), conditions relatives au logiciel et à leur utilisation. Constatant que les banques de terminologie n'ont pas atteint un niveau optimal d'efficacité, il a proposé quelques solutions suggérées par l'évolution générale de l'informatique et de la télématique : adoption du traitement réparti, réseau de banques de tailles et de niveaux différents mais compatibles, recours aux langages de programmation de quatrième génération, interfaces en langue naturelle, etc. Entretemps, selon $M$. Thouin, "la nouvelle condition d'efficacité la plus impérieuse sera l'intégration personnalisée des banques de terminologie avec les autres systèmes existants".

Quant à l'aspect "prospective" du colloque, on peut citer ce passage de la synthèse de Jean Delisle (Université d'Ottawa) : "Les conférenciers qui ont exercé devant nous leur don de clairvoyance (Pierre Laporte, Robert Dubuc, Raymond Frenette et Jean Marguerat) ont regardé dans leur boule de cristal pour essayer d'entrevoir l'avenir. Ils y ont vu que les travaux de néologie s'intensifieront pour suivre l'évolution rapide de la technologie de pointe, que les recherches thématiques se tasseront au profit de la ponctuelle, que l'implantation des terminologies exigera une collaboration accrue des banques et des divers agents producteurs de terminologies, que les banques se ramifieront et uniront leurs efforts et, enfin, que le succès de toute cette vaste opération de francisation et des efforts consentis en terminologie dépend d'une prise de conscience collective."

Claire ChÉnARd-NANTEL ET NOËLLE GUILLOTON 